\title{
Article
}

\section{Operating Vector Solitons with Chirped Sech Pulse Shapes}

\author{
Yan Zhou ${ }^{1, *}$, Keyun Zhang ${ }^{1}$, Meisong Liao ${ }^{2}$, Guoying Zhao ${ }^{3}$ and Yongzheng Fang ${ }^{3}$ \\ 1 School of Science, Shanghai Institute of Technology, Shanghai 201418, China; 216182117@mail.sit.edu.cn \\ 2 Key Laboratory of Materials for High Power Laser, Shanghai Institute of Optics and Fine Mechanics, \\ Chinese Academy of Sciences, Shanghai 201800, China; liaomeisong@siom.ac.cn \\ 3 School of Materials Science and Engineering, Shanghai Institute of Technology, Shanghai 201418, China; \\ zhaogy135@sit.edu.cn (G.Z.); fangyongzheng@sit.edu.cn (Y.F.) \\ * Correspondence: yzhou@sit.edu.cn
}

Citation: Zhou, Y.; Zhang, K.; Liao,

M.; Zhao, G.; Fang, Y. Operating

Vector Solitons with Chirped Sech

Pulse Shapes. Photonics 2022, 9, 143

https://doi.org/10.3390/

photonics 9030143

Received: 5 January 2022

Accepted: 27 February 2022

Published: 28 February 2022

Publisher's Note: MDPI stays neutral with regard to jurisdictional claims in published maps and institutional affiliations.

Copyright: (c) 2022 by the authors. Licensee MDPI, Basel, Switzerland. This article is an open access article distributed under the terms and conditions of the Creative Commons Attribution (CC BY) license (https:// creativecommons.org/licenses/by/ $4.0 /)$.

\begin{abstract}
In this paper, we report the theoretical results about operating vector solitons with chirped sech pulse shapes. In the operation, the shapes of temporal pulses and corresponding optical spectra in orthogonal directions will change, which are based on soliton parameters. When input orthogonal pulses have the same central wavelength of $1064 \mathrm{~nm}$, the shift from the central wavelength always occurs for orthogonal pulses. When input orthogonal pulses have different central wavelengths of $1063 \mathrm{~nm}$ and $1065 \mathrm{~nm}$, output pulse shapes and optical spectra with obvious multiple peak/dip structures can be achieved in orthogonal directions. Our theoretical results are meaningful for the study of vector soliton dynamics and have potential applications in optical communication and optical sensing.
\end{abstract}

Keywords: operation; chirped sech; optical fiber; vector soliton; polarization; group-velocity

\section{Introduction}

Optical soliton in optical fiber has already been studied by many researchers during past decades [1-9]. Commonly, optical soliton can be formed in an anomalous dispersion regime under the balance between group-velocity dispersion and self-phase modulation. With the development of optical soliton theory and relevant experiments, researchers found that optical soliton could also be generated in a normal dispersion regime under the action of many factors, such as gain, group-velocity dispersion, self-phase modulation, saturable absorption, optical spectrum filtering and so on. The study of optical soliton is often performed based on mode-locked fiber laser, because of its compact structure, environmental stability and ease of maintenance [10-15]. Although the single-mode fiber used in constructing fiber laser has circular symmetry, there still exists small linear birefringence (because of fabrication imperfection, bending, heat flow and so on) when optical soliton propagates along the optical fiber. In this situation, the mode of degeneracy in orthogonal polarization directions is broken, and single-mode fiber will support two orthogonally polarized modes. For the case of long-distance optical fiber communication, the optical soliton pulse will suffer from polarization mode dispersion, and the transmitted signal will be distorted. Under this background, the concept of vector soliton was proposed and achieved great developments in theoretical and experimental aspects [16-18]. Vector soliton is robust to linear birefringence and can stably exist in an optical fiber without pulse splitting. Vector soliton can be defined as group-velocity-locking [19-22], polarization-locking [23-25] and polarization rotation [26-28], according to its own characteristics. Theoretically, coupled nonlinear Schrödinger equations [29,30] and coupled Ginzburg-Landau equations [31,32] are usually solved analytically or numerically to analyze the stable vector optical soliton solutions. While experimentally, passively mode-locked fiber laser is often built to study the dynamic properties of vector optical soliton. The orthogonal components of vector soliton can be uncoupled by a polarization beam splitter (PBS) and the real-time dynamics of orthogonal modes can be studied separately [33,34]. 
Until now, there have been many studies about vector solitons achieved by ultra-fast, passively mode-locked fiber lasers. The operating wavelength has been extended to visible light and infrared light regimes, and the vector soliton state has also been enriched. Except for the generation of fiber vector soliton pulse in fiber cavity, the out-cavity operation of vector soliton was also reported in some papers. For example, Wang et al. reported the simulations about the formation of the pseudo-high-order soliton structure through decomposition and recombination of a group-velocity-locked dissipative vector soliton [35]. Our group theoretically studied the operation of polarization- and group-velocity-locked vector solitons with different pulse shapes, such as super-Gaussian pulse shapes [36] and bright-dark pulse pairs [37]. Moreso, theoretical investigations about operating vector bisolitons were conducted [38,39]. In this work, we further explore the operation of vector solitons with chirped sech pulse shapes, which have not been reported until now. Through varying vector soliton parameters in orthogonal polarization directions, the output pulse shapes and optical spectra after recombination in orthogonal directions will demonstrate different properties, which is different from other types of pulse shapes. Our simulation results are useful for the study of vector soliton dynamics and have potential applications in long-distance optical fiber communication and optical fiber sensing.

\section{Model Description}

The theoretical optical fiber system model in our simulation is shown in Figure 1. In this figure, input elliptically polarized vector soliton with an electric amplitude of $A_{1} / A_{2}$ in the vertical/horizontal direction enters into the optical fiber system. The vector soliton is operated through the change of amplitude ratio $\left(A_{2} / A_{1}\right)$, time delay $(\Delta T)$ change in orthogonal directions, pulse chirp $\left(C_{1}\right.$ or $\left.C_{2}\right)$ variation, linear birefringence/phase difference $(\Delta n / \Delta \varphi)$ change and projection angle $(\theta)$ change. SMF is a single-mode fiber. PC is a threepaddle polarization controller that can control the fiber birefringence. According to the relation: $\Delta \varphi_{\text {external }}=\frac{2 \pi \Delta n_{\text {external }}}{\lambda} L$, the externally imposed phase difference $\left(\Delta \varphi_{\text {external }}\right)$ is proportional to the length $(L)$ of SMF. Where $\Delta n_{\text {external }}$ is the externally imposed linear birefringence, $\lambda$ is the wavelength. Col is a fiber collimator that is used for collimating the laser beam. PBS stands for a polarization beam splitter, which can be rotated and change the projection in orthogonal polarization directions ( $x$ and $y$ directions). In vector soliton fiber lasers, the temporal optical pulses often demonstrate sech pulse shapes. In theory, vector soliton solution can be got with the following coupled nonlinear Schrödinger equations:

$$
\begin{aligned}
& \frac{\partial A_{x}}{\partial z}+\beta_{1 x} \frac{\partial A_{x}}{\partial t}+\frac{i \beta_{2}}{2} \frac{\partial^{2} A_{x}}{\partial t^{2}}+\frac{\alpha}{2} A_{x}=i \gamma\left(\left|A_{x}\right|^{2}+\frac{2}{3}\left|A_{y}\right|^{2}\right) A_{x} \\
& \frac{\partial A_{y}}{\partial z}+\beta_{1 y} \frac{\partial A_{y}}{\partial t}+\frac{i \beta_{2}}{2} \frac{\partial^{2} A_{y}}{\partial t^{2}}+\frac{\alpha}{2} A_{y}=i \gamma\left(\left|A_{y}\right|^{2}+\frac{2}{3}\left|A_{x}\right|^{2}\right) A_{y} .
\end{aligned}
$$

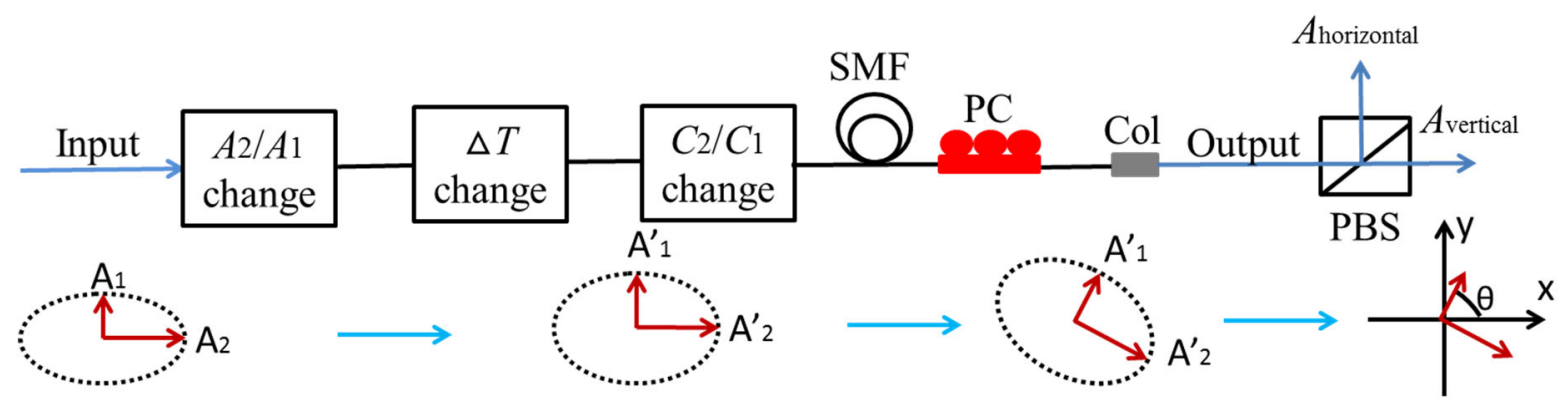

Figure 1. Optical fiber system model in our simulation.

In the above coupled equations, $A_{x}$ and $A_{y}$ are electric fields in orthogonal directions. $\beta_{1 x}$ and $\beta_{1 y}$ are first-order derivatives of the mode propagation constants in orthogonal directions. $\beta_{2}$ is group-velocity dispersion, $\alpha$ is fiber loss, $\gamma$ is fiber nonlinearity, $\mathrm{z}$ is the position variable and $t$ is the time variable. 
Here, we assume input vector solitons in our simulation have sech pulse profiles in orthogonal directions. After modulation of the amplitude ratio, time delay, pulse chirp and phase difference, the electric fields in orthogonal directions after Col and before PBS are expressed as:

$$
\begin{gathered}
A_{1}^{\prime}(t)=A_{1} \operatorname{sech}\left[\frac{1.763(t-\Delta T / 2)}{T_{0}}\right] \exp \left\{-i C_{1}\left[\frac{1.763(t-\Delta T / 2)}{T_{0}}\right]^{2}+i \frac{2 \pi c t}{\lambda_{1}}\right\}, \\
A_{2}^{\prime}(t)=A_{2} \operatorname{sech}\left[\frac{1.763(t+\Delta T / 2)}{T_{0}}\right] \exp \left\{-i C_{2}\left[\frac{1.763(t+\Delta T / 2)}{T_{0}}\right]^{2}+i\left(\frac{2 \pi c t}{\lambda_{2}}+\Delta \phi\right)\right\} .
\end{gathered}
$$

In the above two Equations (2) and (3), $A_{1^{\prime}}(\mathrm{t})$ and $A_{2^{\prime}}(\mathrm{t})$ are time-varying electric field components, $A_{1}$ and $A_{2}$ are corresponding amplitudes. $\lambda_{1}$ and $\lambda_{2}$ are central wavelengths. $C_{1}$ and $C_{2}$ are pulse chirps. $\Delta \varphi$ is the phase difference. The $t$ is time variable, $T_{0}$ is pulse width (same initial pulse width of orthogonal components), $\Delta T$ is time delay, $c$ is the light speed in a vacuum. The projected amplitudes in horizontal and vertical directions after PBS are:

$$
\left[\begin{array}{l}
A_{x}(t) \\
A_{y}(t)
\end{array}\right]=\left[\begin{array}{cc}
\cos \theta & \sin \theta \\
\sin \theta & -\cos \theta
\end{array}\right]\left[\begin{array}{l}
A_{1}^{\prime}(t) \\
A_{2}^{\prime}(t)
\end{array}\right] .
$$

In Equation (4), $A_{x}(t)$ and $A_{y}(t)$ are recombined electric field components in horizontal and vertical directions, respectively. $\theta$ represents the projection angle between the polarization direction of $A_{1^{\prime}}(t)$ and x axis, which is labeled in Figure 1.

$$
\begin{gathered}
I_{i}(t)=\left|A_{i}(t)\right|^{2}(i=x, y), \\
I_{i}(\omega)=\left|\mathcal{F}\left[A_{i}(t)\right]\right|^{2}(i=x, y) .
\end{gathered}
$$

The output pulse shapes after operation can be calculated with Equation (5), and the corresponding optical spectra can be calculated using Equation (6). In these two equations, $I_{i}(t)$ and $I_{i}(\omega)$ are intensities in the time domain and frequency domain, respectively. where $\omega$ is angular frequency, $\mathcal{F}[]$ denotes Fourier transformation.

It should be noted that we simplify the model by assuming the fiber length is much shorter than dispersion length and nonlinear length, and consider the net effects of five different kinds of vector soliton parameters (including amplitude ratio, projection angle, time delay, pulse chirp and phase difference). This assumption has been reported in Ref. [19], and the experimental results confirmed the theoretical assumption. Under our approximation, Equations (2) and (3) are modulated electric components of vector soliton after Col and before PBS. Equation (4) gives polarization to projected electric components after PBS. The simulated pulse shapes and optical spectra can be calculated with Equations (5) and (6), respectively.

\section{Theoretical Results}

\subsection{Equal Central Wavelength}

In this section, we consider the case of equal central wavelength. Figure 2 shows our simulation results when amplitude ratio $\left(A_{2} / A_{1}\right)$ between orthogonal modes is $1 / 1.6$, $1 / 1.3,1 / 1,1.3 / 1,1.6 / 1$, and $\lambda_{1}=\lambda_{2}=1064 \mathrm{~nm}$. Other pulse parameters are: $\theta=0^{\circ}$, $\Delta T=0.5 \mathrm{ps}, C_{1}=C_{2}=3, \Delta \varphi=\pi / 2$. It should be noted that we have assumed $T_{0}=5 \mathrm{ps}$ in all the simulations. From pulse shapes in Figure $2 \mathrm{a}-\mathrm{e}$, we can see the peak intensities of orthogonal pulses have a similar variation as the increase in $A_{2} / A_{1}$. For the optical spectra in Figure 2f-j, a slight wavelength shift from the initial $1064 \mathrm{~nm}$ occurs for both orthogonal modes (red/blue shift for horizontal/vertical mode), accompanied with a similar peak intensity variation trend compared with corresponding pulse shapes. The relatively large optical spectrum bandwidths $(\sim 4 \mathrm{~nm})$ of orthogonal components are mainly induced by non-zero chirp parameters, and the $3-\mathrm{dB}$ bandwidths can be further increased with larger pulse chirps in orthogonal directions. 

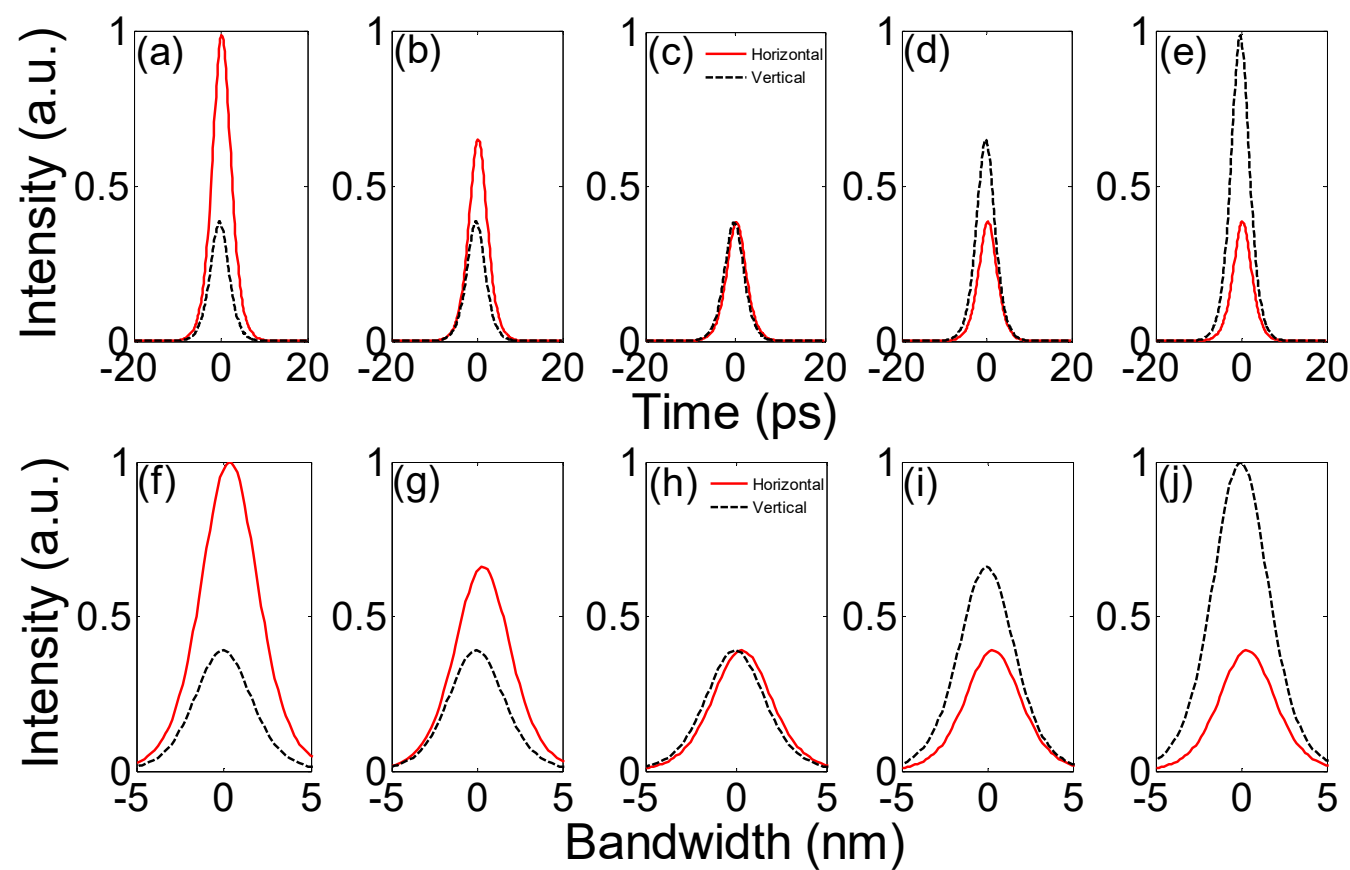

Figure 2. Theoretical results after polarization splitting when the amplitude ratio between orthogonal modes is changed and $\lambda_{1}=\lambda_{2}=1064 \mathrm{~nm}$.

Figure 3 shows our simulation results when projection angle $(\theta)$ is $15^{\circ}, 30^{\circ}, 45^{\circ}$, $60^{\circ}, 75^{\circ}$, and $\lambda_{1}=\lambda_{2}=1064 \mathrm{~nm}$. Other pulse parameters are: $A_{2} / A_{1}=1 / 1, \Delta T=1 \mathrm{ps}$, $C_{1}=C_{2}=3, \Delta \varphi=\pi / 2$. In Figure 3a-e, the peak intensities of orthogonal pulses have no obvious changes when $\theta$ increases. However, the time delay between orthogonal pulses slightly varies with the change of $\theta$. When $\theta=45^{\circ}$, the orthogonal pulses are overlapped, and the time delay will increase when $\theta$ is close to $0^{\circ}$ or $90^{\circ}$. In the corresponding optical spectra of Figure $3 \mathrm{f}-\mathrm{j}$, the central wavelengths of orthogonal pulses shift from the initial $1064 \mathrm{~nm}$, and the offset length changes with $\theta$. The orthogonal pulses' optical spectra do not coincide with each other, even when $\theta=45^{\circ}$.
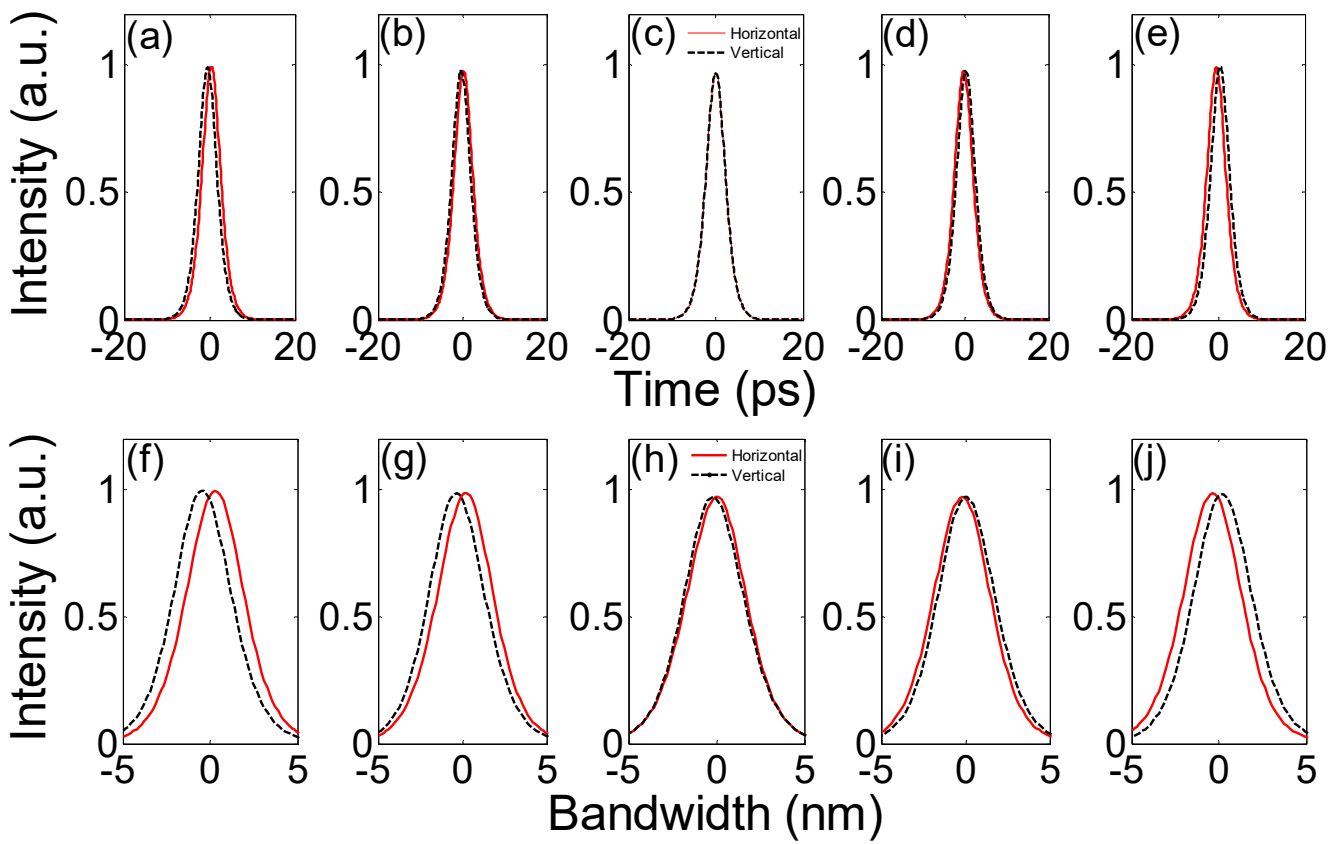

Figure 3. Theoretical results after polarization splitting when the projection angle is changed and $\lambda_{1}=\lambda_{2}=1064 \mathrm{~nm}$. 
Figure 4 shows our simulation results when the time delay $(\triangle T)$ is 2 ps, 4 ps, 6 ps, 8 ps, $10 \mathrm{ps}$, and $\lambda_{1}=\lambda_{2}=1064 \mathrm{~nm}$. Other pulse parameters are: $A_{2} / A_{1}=1 / 1, \theta=5^{\circ}, C_{1}=C_{2}=3$, $\Delta \varphi=\pi / 2$. From the pulse shapes in Figure $4 \mathrm{a}-\mathrm{e}$, we can see with the increase of $\Delta T$, the temporal delay of output pulse peaks in orthogonal directions will also increase, and the peak intensities and pulse widths have no obvious changes. While from the optical spectra in Figure $4 \mathrm{f}-\mathrm{j}$, it is obvious that the central wavelength difference of output orthogonal pulses increases with the increased $\Delta T$ value. The central wavelength difference is $\sim 7 \mathrm{~nm}$ when $\Delta T=10 \mathrm{ps}$.
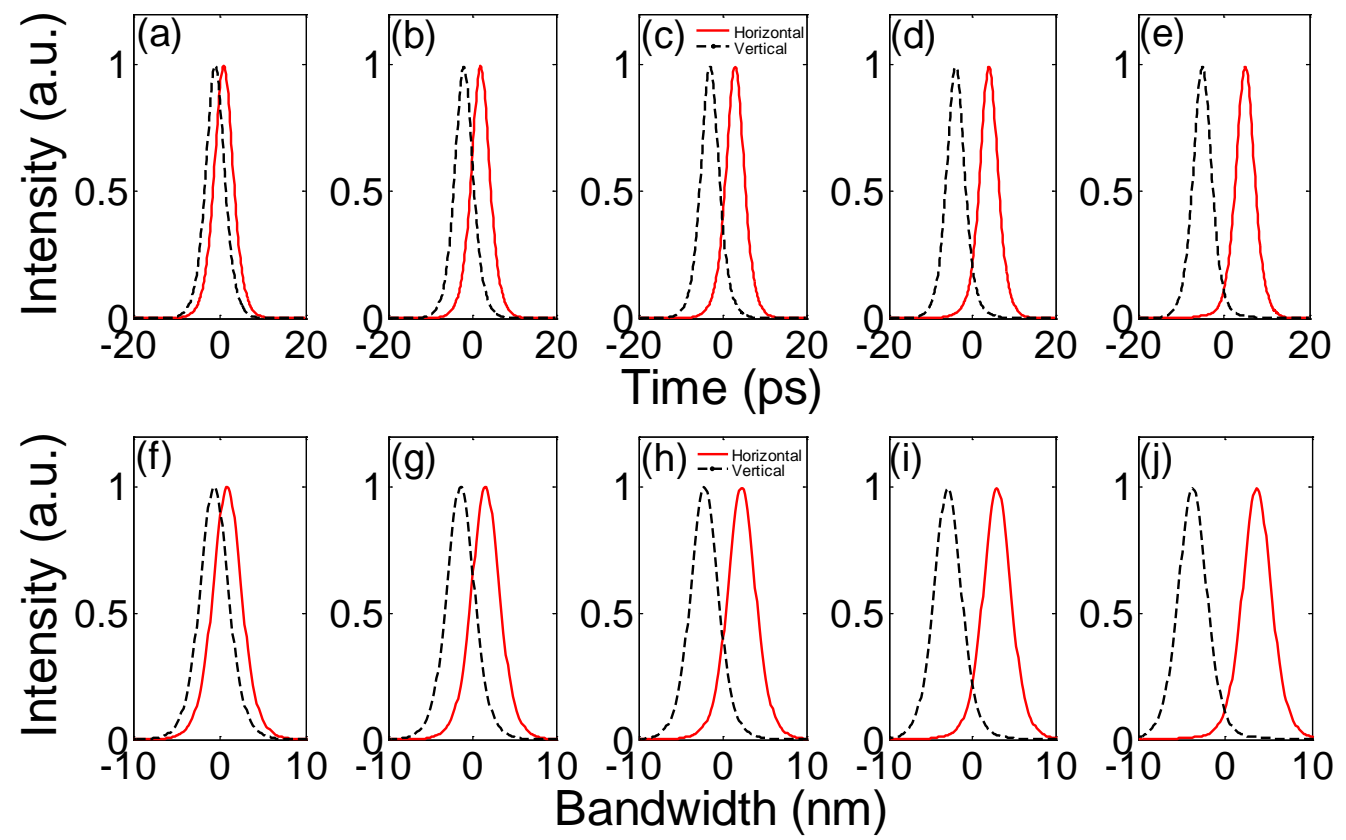

Figure 4. Theoretical results after polarization splitting when the time delay is changed and $\lambda_{1}=\lambda_{2}=1064 \mathrm{~nm}$.

Figure 5 shows our simulation results when the pulse chirp $\left(C_{1}=C_{2}\right)$ is $3,6,9,12$, 15 , and $\lambda_{1}=\lambda_{2}=1064 \mathrm{~nm}$. Other pulse parameters are: $A_{2} / A_{1}=1 / 1, \theta=5^{\circ}, \Delta T=1 \mathrm{ps}$, $\Delta \varphi=\pi / 2$. From Figure $5 \mathrm{a}-\mathrm{e}$, we can see the pulse shapes in orthogonal polarization directions have no obvious changes with the increase in pulse chirp. However, in Figure $5 \mathrm{f}-\mathrm{j}$, the corresponding optical spectra will change with increased chirp value. The peak intensities or pulse widths of orthogonal modes will decrease or increase when the chirp increases from 3 to 15. Besides, the optical spectra of orthogonal pulses will become rough when the pulse chirp is 12 or 15, which means the ripples are caused by a relatively large chirp value. Moreso, the central wavelength shift still exists in this situation.

Figure 6 shows our simulation results when phase difference $(\Delta \varphi)$ is $\pi / 7,2 \pi / 7,3 \pi / 7$, $4 \pi / 7,5 \pi / 7$, and $\lambda_{1}=\lambda_{2}=1064 \mathrm{~nm}$. Other pulse parameters are: $A_{2} / A_{1}=1 / 1, \theta=5^{\circ}$, $\Delta T=1$ ps, $C_{1}=C_{2}=3$. Figure 6 a-e show that the peak intensity of horizontal/vertical pulse will decrease/increase with an increased phase difference. While in Figure $6 f-j$, the peak intensity of the horizontal/vertical pulse spectra have the same variation trend compared with Figure 6a-e. The central wavelength difference of orthogonal pulses is $\sim 1 \mathrm{~nm}$, regardless of the initial $1064 \mathrm{~nm}$ central wavelengths. 

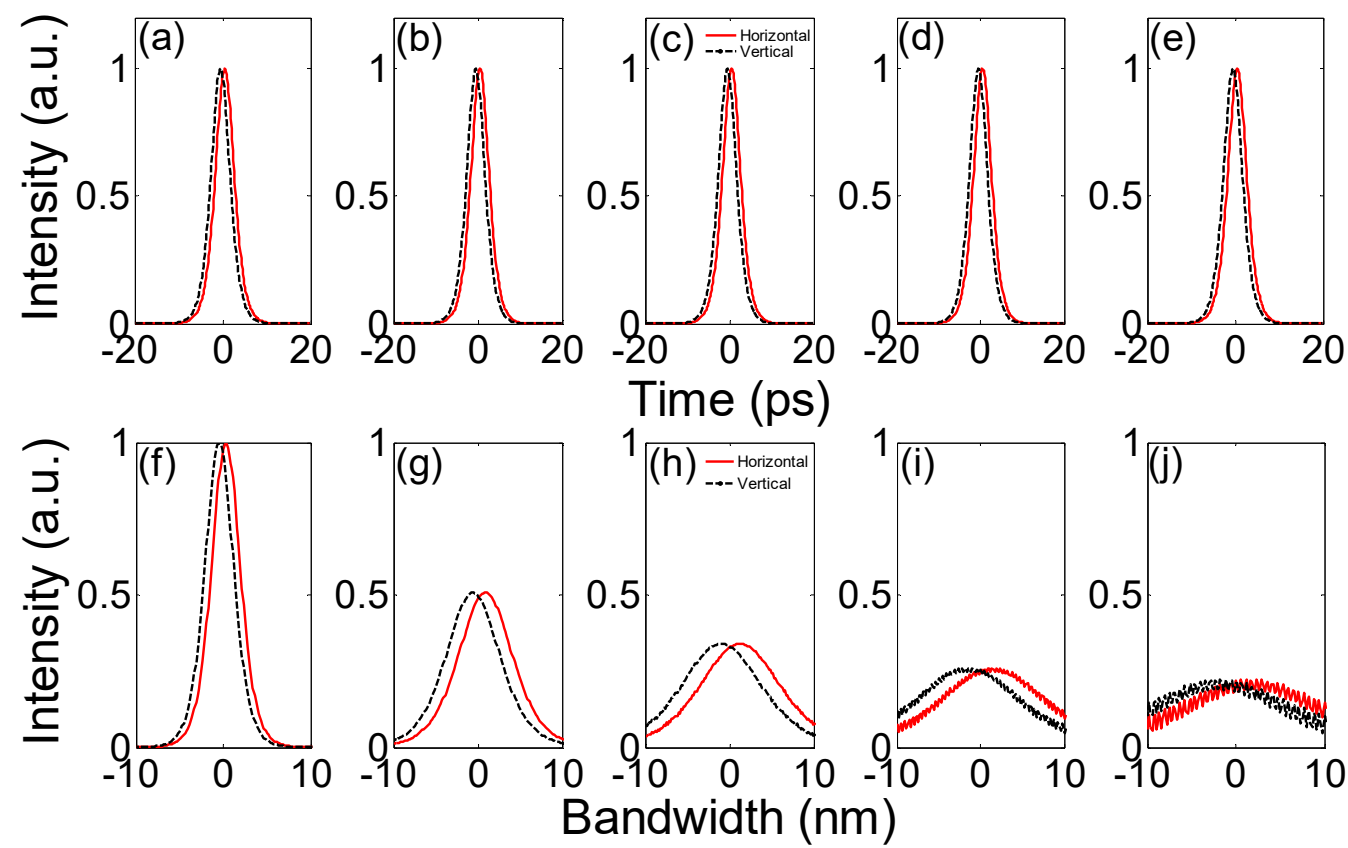

Figure 5. Theoretical results after polarization splitting when the pulse chirp is changed and $\lambda_{1}=\lambda_{2}=1064 \mathrm{~nm}$.
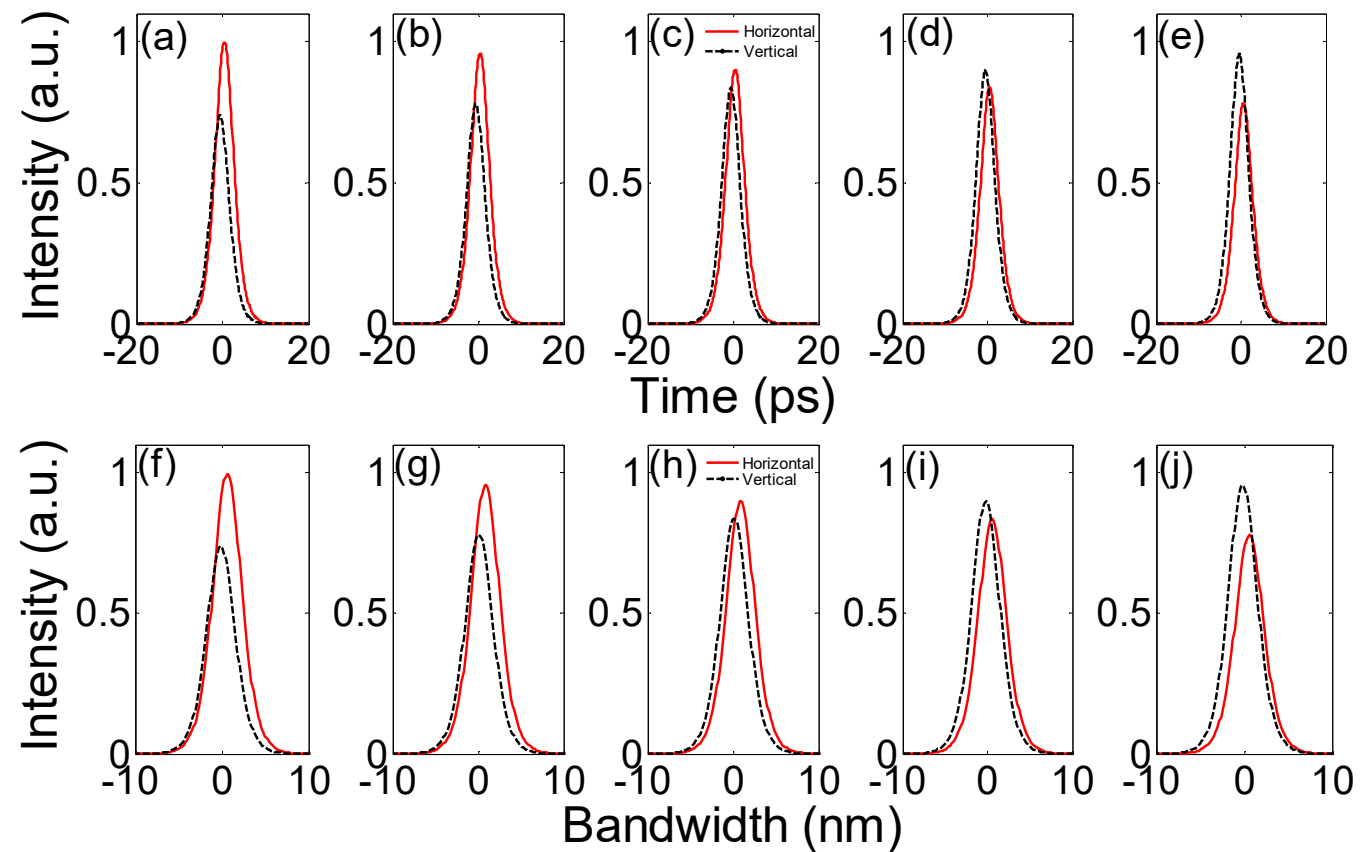

Bandwidth (nm)

Figure 6. Theoretical results after polarization splitting when the phase difference is changed and $\lambda_{1}=\lambda_{2}=1064 \mathrm{~nm}$.

\subsection{Different Central Wavelengths}

In this section, we consider the case of different central wavelengths. Figure 7 shows our simulation results when the amplitude ratio $\left(A_{2} / A_{1}\right)$ between orthogonal modes is $1 / 1.6,1 / 1.3,1 / 1,1.3 / 1,1.6 / 1$, and $\lambda_{1}=1063 \mathrm{~nm}, \lambda_{2}=1065 \mathrm{~nm}$. Other pulse parameters are: $\theta=0^{\circ}, \triangle T=0.5 \mathrm{ps}, C_{1}=C_{2}=3, \triangle \varphi=0$. The pulse shapes in Figure 7a-e behave similarly to Figure $2 \mathrm{a}-\mathrm{e}$, even though with different central wavelengths and phase differences. However, the optical spectra of orthogonal pulses in Figure $7 \mathrm{f}-\mathrm{j}$ are obviously separate from each other, compared with Figure $2 \mathrm{f}-\mathrm{j}$. The reason is that the initial $2 \mathrm{~nm}$ central wavelength difference enlarges the central wavelength difference between the horizontal component 
and vertical component. Similarly, to Figure $2 \mathrm{f}-\mathrm{j}$, the $3-\mathrm{dB}$ optical spectra bandwidths of orthogonal pulses in Figure $7 \mathrm{f}-\mathrm{j}$ have no obvious changes because of unchanged chirps.
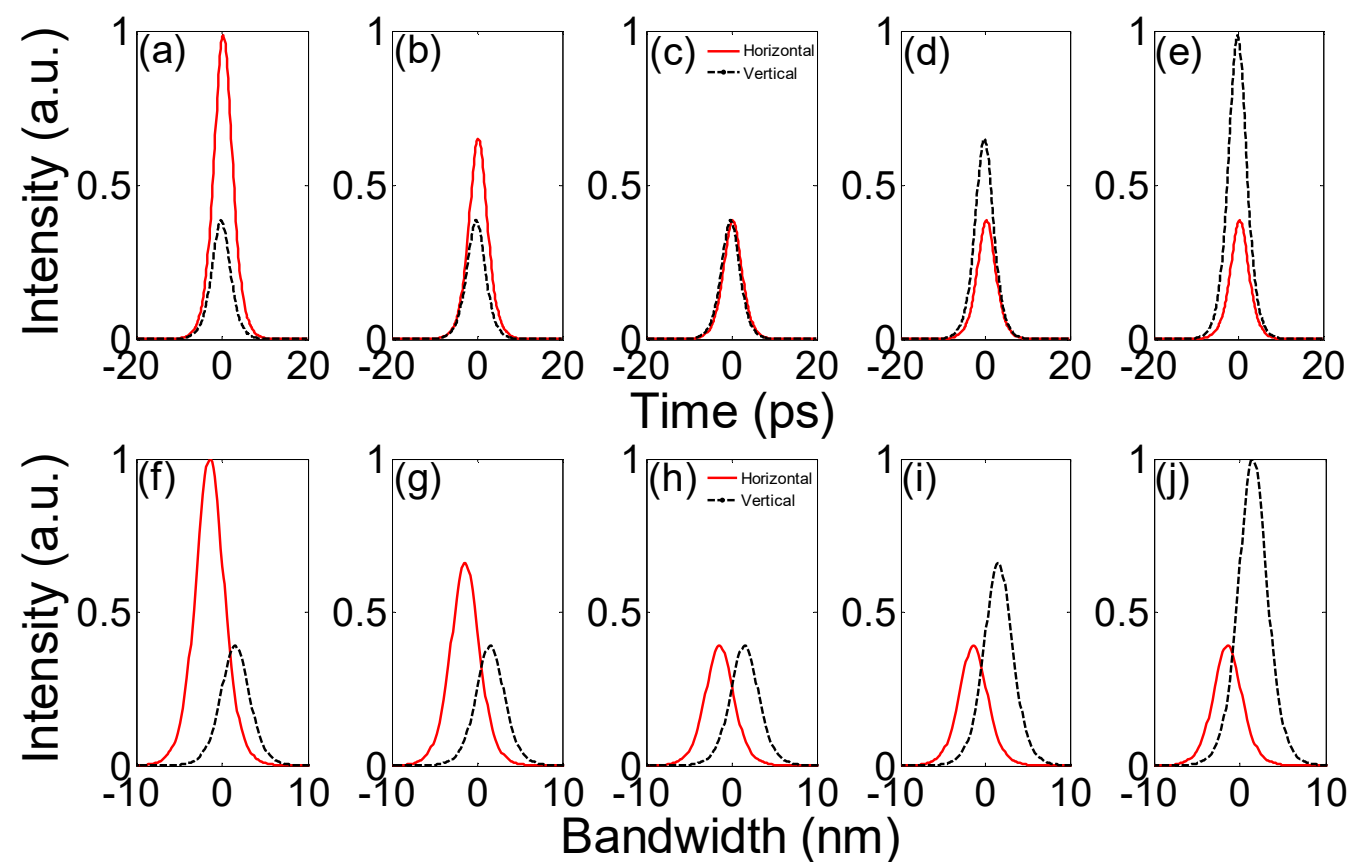

Figure 7. Theoretical results after polarization splitting when amplitude ratio between orthogonal modes is changed and $\lambda_{1}=1063 \mathrm{~nm}, \lambda_{2}=1065 \mathrm{~nm}$.

Figure 8 shows our simulation results when the projection angle $(\theta)$ is $15^{\circ}, 30^{\circ}, 45^{\circ}$, $60^{\circ}, 75^{\circ}$, and $\lambda_{1}=1063 \mathrm{~nm}, \lambda_{2}=1065 \mathrm{~nm}$. Other pulse parameters are: $A_{2} / A_{1}=1 / 1$, $\Delta T=1 \mathrm{ps}, C_{1}=C_{2}=3, \Delta \varphi=0$. In Figure 8a-e, we can see that temporal splitting occurs for both orthogonal pulses. The peak intensities of the main peaks in orthogonal directions will reach maximum when $\theta=45^{\circ}$ and will decrease when $\theta$ is close to $0^{\circ}$ or $90^{\circ}$. Besides, the oscillation depth of horizontal/vertical pulse nearly covers the whole pulse waveform when $\theta=45^{\circ}$. In Figure 8f-j, both the central wavelength shift and optical spectrum splitting occur for horizontal/vertical pulse, which means multi-wavelength vector solitons are generated in this situation. When $\theta=45^{\circ}$, the envelopes of orthogonal modes are almost overlapped, but their peak/dip details are still different.

Figure 9 shows our simulation results when the time delay $(\Delta T)$ is $2 \mathrm{ps}, 4 \mathrm{ps}, 6 \mathrm{ps}$, $8 \mathrm{ps}, 10 \mathrm{ps}$, and $\lambda_{1}=1063 \mathrm{~nm}, \lambda_{2}=1065 \mathrm{~nm}$. Other pulse parameters are: $A_{2} / A_{1}=1 / 1$, $\theta=5^{\circ}, C_{1}=C_{2}=3, \Delta \varphi=0$. In Figure 9a-e, the orthogonal pulses begin to separate with each other when $\Delta T$ starts to increase, accompanied by the appearance of temporal side peaks. When $\Delta T$ reaches $6 \mathrm{ps}$, the orthogonal pulses become smooth and there are only some small oscillations at the pedestals. In Figure $9 \mathrm{f}-\mathrm{j}$, the optical spectra of orthogonal pulses are always not smooth with the increase in $\Delta T$. The central wavelength of horizontal mode is obviously smaller/larger than vertical mode when $\Delta T$ is smaller/larger than $4 \mathrm{ps}$. Additionally, the central wavelength difference of orthogonal pulses increases with increased $\Delta T$ when the time delay is larger than $4 \mathrm{ps.}$ 

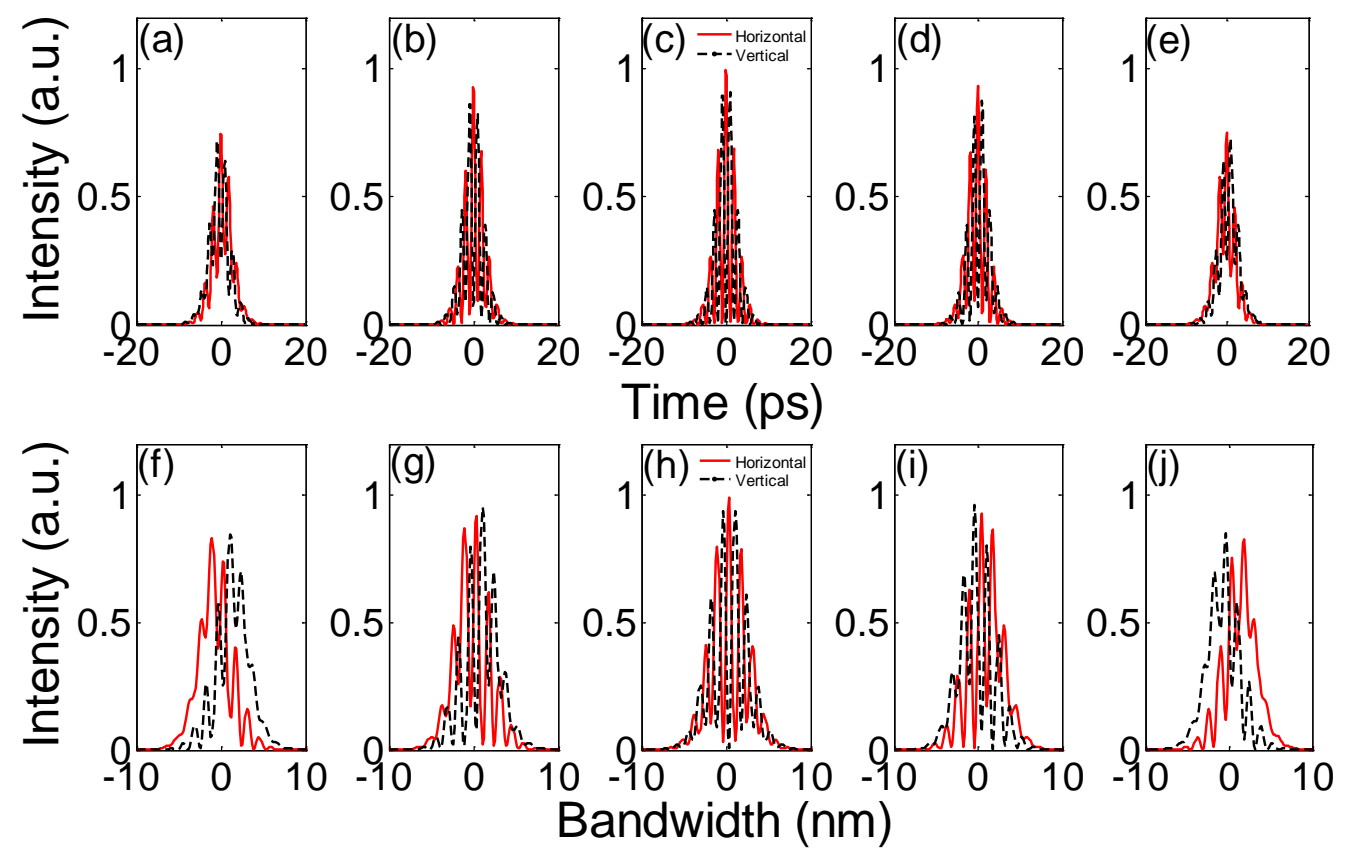

Figure 8. Theoretical results after polarization splitting when projection angle is changed and $\lambda_{1}=1063 \mathrm{~nm}, \lambda_{2}=1065 \mathrm{~nm}$.
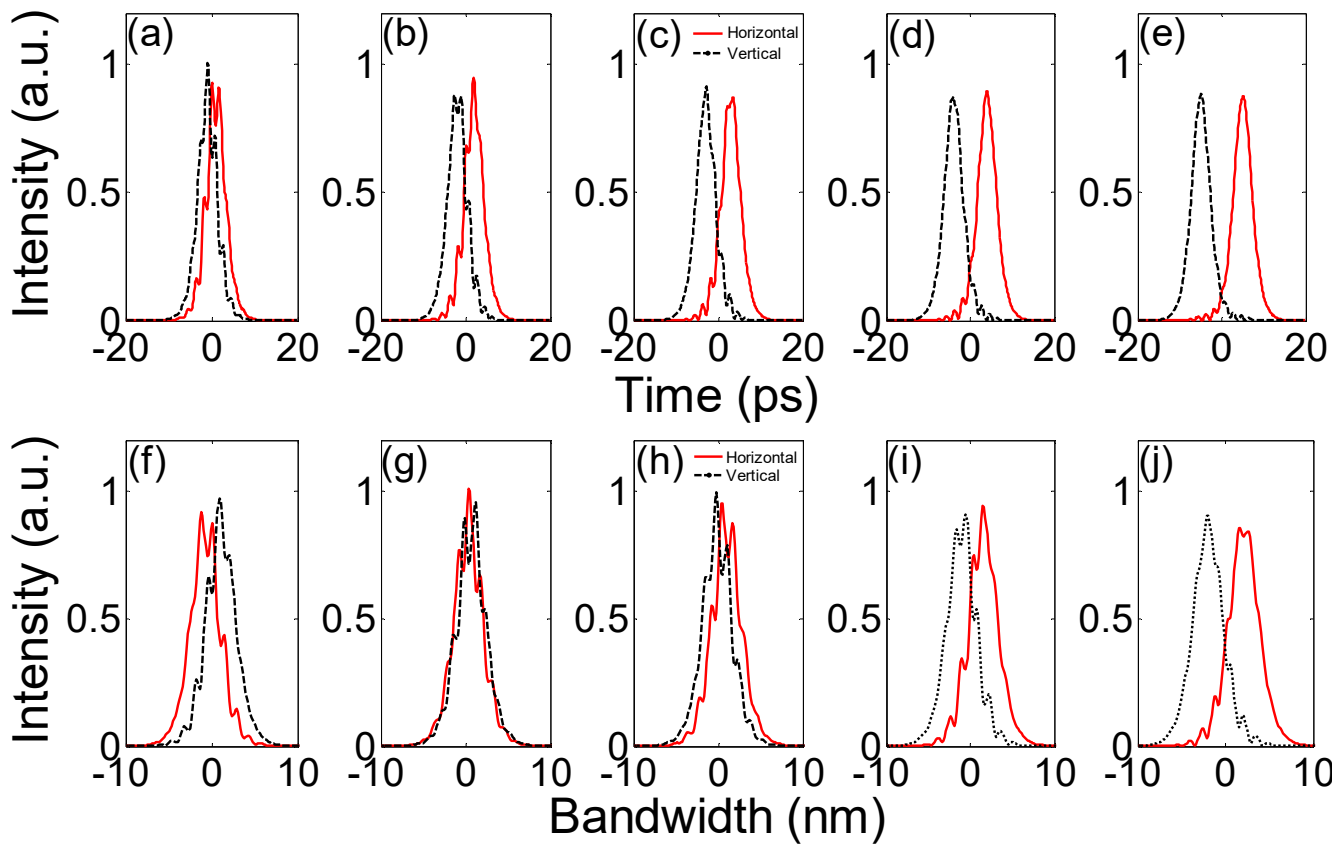

Figure 9. Theoretical results after polarization splitting when the time delay is changed and $\lambda_{1}=1063 \mathrm{~nm}, \lambda_{2}=1065 \mathrm{~nm}$.

Figure 10 shows our simulation results when chirp $\left(C_{1}=C_{2}\right)$ is $3,6,9,12,15$, and $\lambda_{1}=1063 \mathrm{~nm}, \lambda_{2}=1065 \mathrm{~nm}$. Other pulse parameters are: $A_{2} / A_{1}=1 / 1, \theta=5^{\circ}, \Delta T=1 \mathrm{ps}$, $\Delta \varphi=0$. In Figure 10a-e, the peak intensities of orthogonal pulses have no obvious changes with the increase in pulse chirp, and side peaks always exist for both orthogonal modes. In Figure 10f-j, the peak intensities/bandwidths of orthogonal pulses will decrease/increase when the pulse chirp increases. Besides, the adjacent peak-dip intensity difference in the horizontal direction or vertical direction will decrease with the increase in pulse chirp. The ripples in Figure 10i,j are caused by a relatively large chirp value. 

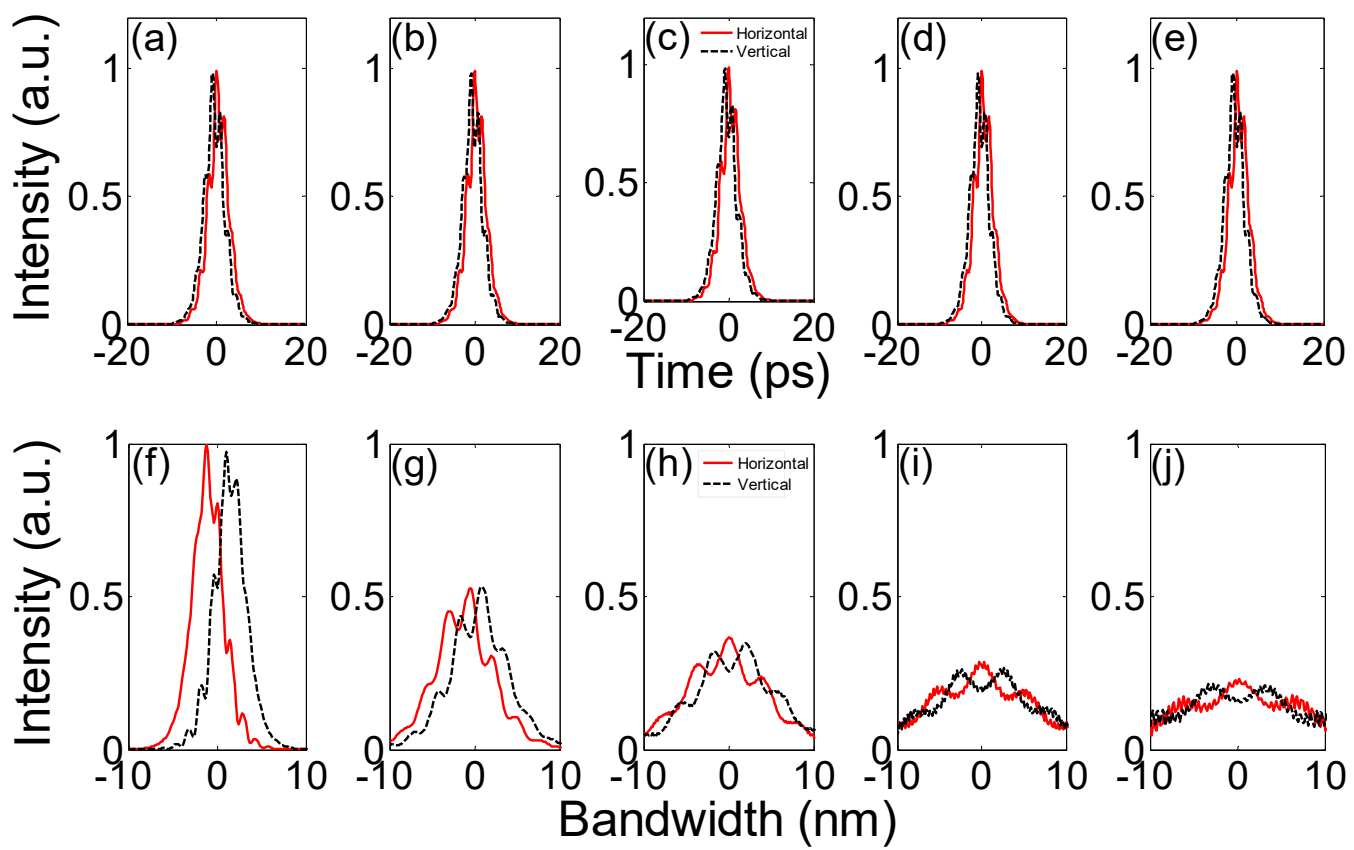

Figure 10. Theoretical results after polarization splitting when pulse chirp is changed and $\lambda_{1}=1063 \mathrm{~nm}, \lambda_{2}=1065 \mathrm{~nm}$.

Figure 11 shows our simulation results when phase difference $(\Delta \varphi)$ is $\pi / 7,2 \pi / 7,3 \pi / 7$, $4 \pi / 7,5 \pi / 7$, and $\lambda_{1}=1063 \mathrm{~nm}, \lambda_{2}=1065 \mathrm{~nm}$. Other pulse parameters are: $A_{2} / A_{1}=1 / 1$, $\theta=5^{\circ}, \Delta T=1 \mathrm{ps}, C_{1}=C_{2}=3$. In Figure 11a-e, the peak intensities of orthogonal pulses are almost unchanged with the increase in phase difference, accompanied by slight pulse splitting, and the peak intensities of both orthogonal pulses are the same, which is different from Figure 6a-e. In Figure 11f-j, the optical spectra peak intensities of orthogonal pulses are also unchanged when phase difference increases. Horizontal/vertical pulse is always at 1063/1065 nm and side peaks exist for both orthogonal pulses.
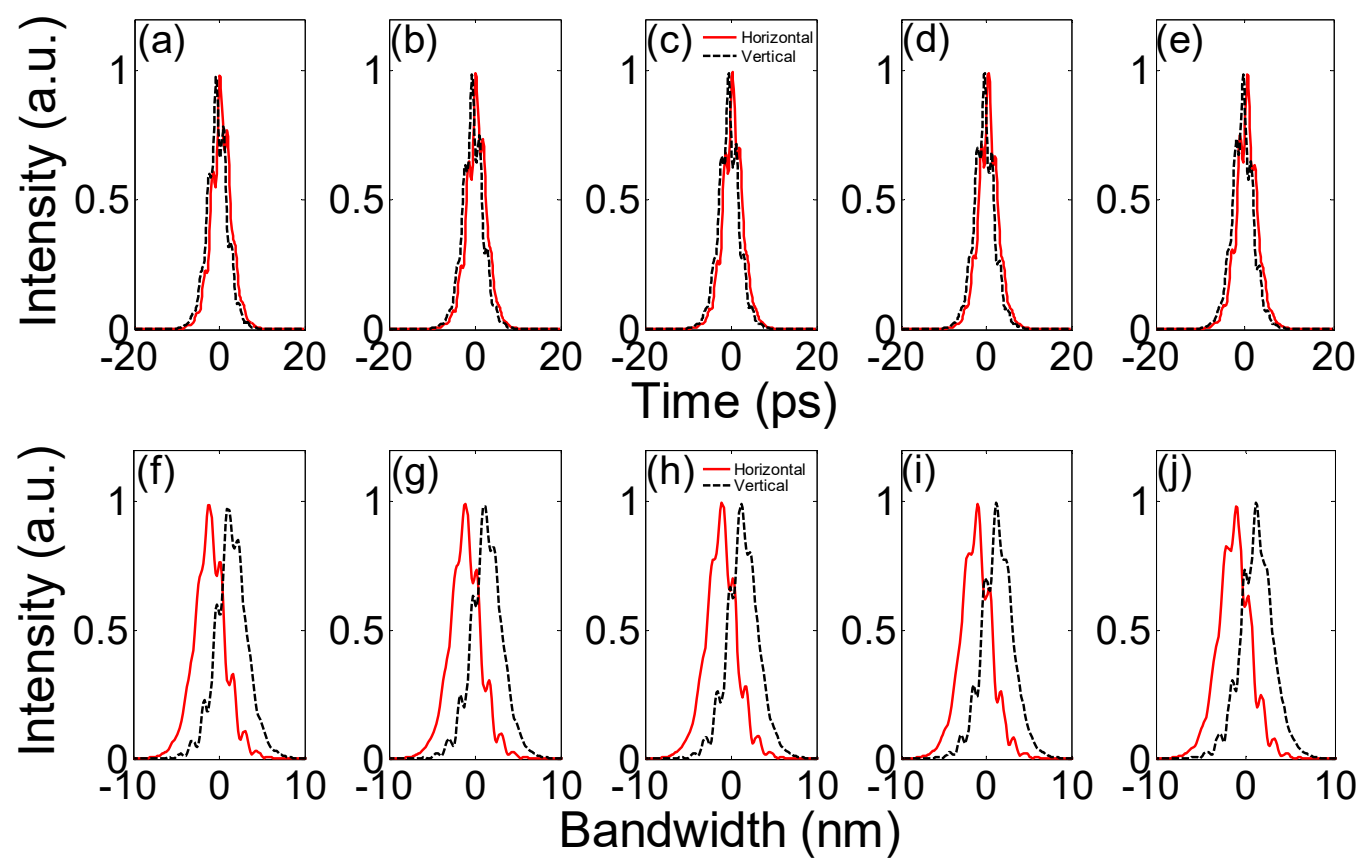

Figure 11. Theoretical results after polarization splitting when the phase difference is changed and $\lambda_{1}=\lambda_{2}=1064 \mathrm{~nm}$. 


\section{Conclusions}

We simulated the operation of vector solitons with chirped sech pulse shapes. Through the change of vector soliton parameters (including amplitude ratio, projection angle, time delay, pulse chirp and phase difference) when input orthogonal pulses have the same or different central wavelengths, output orthogonally polarized pulses would demonstrate different characteristics in the time domain and frequency domain. Our simulations explored the dynamics of operating chirped sech vector solitons, which are beneficial to the study of vector soliton dynamics and relevant fields that employ vector optical solitons.

Author Contributions: Conceptualization, Y.Z.; methodology, Y.Z. and K.Z.; validation, Y.Z. and K.Z.; writing—original draft preparation, Y.Z.; writing—review and editing, G.Z., M.L. and Y.F.; visualization, Y.Z.; supervision, G.Z., M.L. and Y.F.; funding acquisition, Y.Z., M.L. and Y.F. All authors have read and agreed to the published version of the manuscript.

Funding: This research was funded by the National Natural Science Foundation of China (NSFC), grant numbers 5217021588, 62105208 and 51972317; Shanghai Sailing Program, grant number 20YF1447500.

Data Availability Statement: Not applicable.

Conflicts of Interest: The authors declare no conflict of interest.

\section{References}

1. Kaup, D.J.; EI-Reedy, J.; Malomed, B.A. Effect of a chirp on soliton production. Phys. Rev. E 1994, 50, 1635. [CrossRef]

2. Triki, H.; Zhou, Q.; Biswas, A.; Liu, W.; Yildirim, Y.; Alshehri, H.M.; Belic, M.R. Chirped optical solitons having polynomial law of nonlinear refractive index with self-steepening and nonlinear dispersion. Phys. Lett. A 2021, 417, 127698. [CrossRef]

3. Yang, J.; Huang, Z.H.; Zhu, Y.; Zhou, Q.; Li, J.T.; Sun, Y.Z.; Alshehri, H.M.; Biswas, A. Soliton-soliton interaction and its influence on soliton amplitude and period. Results Phys. 2021, 30, 104831. [CrossRef]

4. Zayed, E.M.E.; Alngar, M.E.M.; Biswas, A.; Ekici, M.; Arshed, S.; Alzahrani, A.K.; Belic, M.R. Solitons in nonlinear directional couplers with optical metamaterials by unified Riccati equation approach. Optik 2021, 241, 167244. [CrossRef]

5. Sorokin, A.A.; Anashkina, E.A.; Corney, J.F.; Bobrovs, V.; Leuchs, G.; Andrianov, A.V. Numerical simulations on polarization quantum noise squeezing for ultrashort solitons in optical fiber with enlarged mode field area. Photonics 2021, 8, 228. [CrossRef]

6. Wang, X.J.; Zhang, C.X.; Wang, Z.H.; Liu, J.; Zhang, H.; Fan, D.Y. Dynamic behaviors of multiple-soliton pulsation in an L-band passively mode-locked fiber laser with anomalous dispersion. Chaos 2021, 31, 063122. [CrossRef]

7. Zhu, X.R.; Zhang, B.; Zhao, D.S.; Yang, L.Y.; Liu, S.L.; Hou, J. Pulse evolution and multi-pulse state of coherently coupled polarization domain walls in a fiber ring laser. Opt. Express 2021, 29, 30558-30566. [CrossRef]

8. Liu, X.Y.; Zhang, H.X.; Liu, W.J. The dynamic characteristics of pure-quartic solitons and soliton molecules. Appl. Math. Model. 2022, 102, 305-312. [CrossRef]

9. Yan, X.W.; Chen, Y. Soliton interaction of a generalized nonlinear Schrodinger equation in an optical fiber. Appl. Math. Lett. 2022, 125, 107737. [CrossRef]

10. Huang, L.; Zhang, Y.S.; Cui, Y.D.; Qiu, J.R.; Liu, X.M. Microfiber-assisted gigahertz harmonic mode-locking in ultrafast fiber laser. Opt. Lett. 2020, 45, 4678-4681. [CrossRef]

11. Yang, K.; Li, T.J.; Li, X.D.; Chen, J.X.; Liu, M.; Cui, H.; Luo, A.P.; Xu, W.C.; Luo, Z.C. Mutually induced soliton polarization instability in a bidirectional ultrafast fiber laser. Opt. Lett. 2021, 46, 4848-4851. [CrossRef] [PubMed]

12. Ma, X.H.; Chen, W.; Tong, L.; Liu, S.Q.; Dai, W.W.; Ye, S.S.; Zheng, Z.Q.; Wang, Y.Y.; Zhou, Y.; Zhang, W.; et al. In $2 S_{3}-$ based saturable absorber for passively harmonic mode-locking in $2 \mu \mathrm{m}$ region. Opt. Laser Technol. 2022, 145, 107476. [CrossRef]

13. Lee, J.; Kwon, S.; Zhao, L.M.; Lee, J.H. Investigation into the impact of the recovery time of a saturable absorber for stable dissipative soliton generation in Yb-doped fiber lasers. Opt. Express 2021, 29, 21978-21991.

14. Guo, J.; Hu, X.; Ma, J.; Zhao, L.M.; Shen, D.Y.; Tang, D.Y. Anti-dark solitons in a single mode fiber laser. Phys. Lett. A 2021, 395, 127226. [CrossRef]

15. Dai, L.L.; Huang, Z.N.; Huang, Q.Q.; Zhao, C.; Rozhin, A.; Sergeyev, S.; Al Araimi, M.; Mou, C.B. Carbon nanotube mode-locked fiber lasers: Recent progress and perspectives. Nanophotonics 2021, 10, 749-775. [CrossRef]

16. Menyuk, C.R. Stability of solitons in birefringent optical fibers. I: Equal propagation amplitudes. Opt. Lett. 1987, 12, 614-616. [CrossRef]

17. Menyuk, C.R. Stability of solitons in birefringent optical fibers. II. Arbitrary amplitudes. J. Opt. Soc. Am. B 1988, 5, 392. [CrossRef]

18. Christodoulides, D.N.; Joseph, R.I. Vector solitons in birefringent nonlinear dispersive media. Opt. Lett. 1988, 13, 53-55. [CrossRef]

19. Jin, X.X.; Wu, Z.C.; Li, L.; Zhang, Q.; Tang, D.Y.; Shen, D.Y.; Fu, S.N.; Liu, D.M.; Zhao, L.M. Manipulation of group-velocity-locked vector solitons from fiber lasers. IEEE Photonics J. 2016, 8, 1501206. [CrossRef] 
20. Luo, Y.Y.; Cheng, J.W.; Liu, B.W.; Sun, Q.Z.; Li, L.; Fu, S.N.; Tang, D.Y.; Zhao, L.M.; Liu, D.M. Group-velocity-locked vector soliton molecules in fiber lasers. Sci. Rep. 2017, 7, 2369. [CrossRef]

21. Xia, R.; Luo, Y.Y.; Shum, P.P.; Xiang, Y.; Ni, W.J.; Liu, Y.S.; Lam, H.Q.; Tang, D.Y.; Tang, X.H. Vectorial nature in nonlinear multimode interference based ultrafast fiber lasers. IEEE Photonics J. 2020, 12, 1501310. [CrossRef]

22. Wang, J.Z.; Jin, L.; Xie, S.Z.; Wang, R.Y.; Zhang, H.; Xu, Y.T.; Zhao, X.; Li, Y.; Ma, X.H. Vector dynamics of ultrafast cylindrical vector beams in a mode-locked fiber laser. Chin. Opt. Lett. 2021, 19, 111903. [CrossRef]

23. Wu, Z.C.; Wei, Q.; Huang, P.; Luo, D.P.; Zhang, X.L.; Fu, S.N.; Zhao, L.M.; Liu, D.M.; Shum, P.; Huang, T.Y. Single-axis soliton molecule and multiple solitons generation from a vector fiber laser. Opt. Express 2020, 28, 5212-5220. [CrossRef] [PubMed]

24. Wu, Z.C.; Zhan, B.Y.; Li, L.; Huang, T.Y.; Wei, Q.; Zhang, D.Z.; Shum, P.P. Vector soliton generation from a compact all-polarizationmaintaining fiber laser. Laser Phys. Lett. 2021, 18, 025103. [CrossRef]

25. Li, L.; Pang, L.H.; Wang, R.F.; Zhang, X.G.; Hui, Z.Q.; Han, D.D.; Zhao, F.; Liu, W.J. Ternary transition metal dichalcogenides for high power vector dissipative soliton ultrafast fiber laser. Laser Photonics Rev. 2021, 16, 2100255. [CrossRef]

26. Luo, X.; Tuan, T.H.; Saini, T.S.; Nguyen, H.P.T.; Suzuki, T.; Ohishi, O. Group velocity locked vector soliton and polarization rotation vector soliton generation in a highly birefringent fiber laser. Jpn. J. Appl. Phys. 2019, 58, 020910. [CrossRef]

27. Liu, W.; Wang, W.L.; He, B.; Chen, X.W.; Hu, X.; Guo, Y.K.; Xu, Y.; Wei, X.M. Vector soliton dynamics in a high-repetition-rate fiber laser. Opt. Express 2021, 29, 12049-12065.

28. Zhao, K.J.; Gao, C.X.; Xiao, X.S.; Yang, C.X. Vector quartic solitons in birefringent fibers. Opt. Lett. 2021, 46, 761-764. [CrossRef]

29. Wang, L.L.; Luan, Z.T.; Zhou, Q.; Biswas, A.; Alzahrani, A.K.; Liu, W. Bright soliton solutions of the (2+1)-dimensional generalized coupled nonlinear Schrodinger equation with the four-wave mixing term. Nonlinear Dyn. 2021, 104, 2613-2620. [CrossRef]

30. Stalin, S.; Ramakrishnan, R.; Lakshmanan, M. Nondegenerate bright solitons in coupled nonlinear Schrödinger systems: Recent developments on optical vector solitons. Photonics 2021, 8, 258. [CrossRef]

31. Hu, X.; Guo, J.; Zhao, L.M.; Ma, J.; Tang, D.Y. Dark-bright soliton trapping in a fiber laser. Opt. Lett. 2021, 46, 1105-1108. [CrossRef] [PubMed]

32. Klein, A.; Meir, S.; Duadi, H.; Govindarajan, A.; Fridman, M. Polarization dynamics of ultrafast solitons. Opt. Express 2021, 29, 18512-18522. [CrossRef] [PubMed]

33. Zhao, K.J.; Gao, C.X.; Xiao, X.S.; Yang, C.X. Real-time collision dynamics of vector solitons in a fiber laser. Photon. Res. 2021, 9 , 289-298. [CrossRef]

34. Du, W.X.; Li, H.P.; Li, J.W.; Wang, Z.; Zhang, Z.Y.; Zhang, S.J.; Liu, Y. Real-time observation of pulsating period-doubled vector solitons in a passively mode-locked fiber laser. Opt. Express 2021, 29, 14101-14111. [CrossRef] [PubMed]

35. Wang, X.; Li, L.; Geng, Y.; Wang, H.X.; Su, L.; Zhao, L.M. Decomposition of group-velocity-locked-vector-dissipative solitons and formation of the high-order soliton structure by the product of their recombination. Appl. Opt. 2018, 57, 746-751. [CrossRef] [PubMed]

36. Zhou, Y.; Li, Y.F.; Zhang, R.L.; Wang, T.X.; Bi, W.J.; Li, X.; Kuan, P.W.; Fang, Y.Z.; Liao, M.S. Generation of pseudo-high-order polarization-locked vector solitons with super-Gaussian pulses. Optik 2019, 194, 163132. [CrossRef]

37. Zhou, Y.; Li, Y.F.; Li, X.; Liao, M.S.; Hou, J.S.; Fang, Y.Z. Pulse shaping of bright-dark vector soliton pair. Chin. Phys. B 2020, 29 , 054202. [CrossRef]

38. Zhou, Y.; Lin, X.Y.; Liao, M.S.; Zhao, G.Y.; Fang, Y.Z. Modulating self-similar vector bisolitons. Optik 2021, 244, 167616. [CrossRef]

39. Zhou, Y.; Lin, X.Y.; Liao, M.S.; Zhao, G.Y.; Fang, Y.Z. Polarization manipulation of bright-dark vector bisolitons. Chin. Phys. B 2021, 30, 034208. [CrossRef] 